Original

\title{
Resultados a largo plazo del estudio multicéntrico fase III del tratamiento de la incontinencia de orina post prostatectomia con un sling masculino ajustable: seguimiento mínimo 3 años
}

\author{
Salomon V. Romano*, Sergio E. Metrebian**, Fernando Vaz***, Valter Muller***, \\ Carlos A. Levi D’Ancona****, Eugenio A. Costa de Souza*****, Fabio Nakamura****** \\ *Urology Department, Hospital Durand. Buenos Aires, Argentina \\ **Urology Department, Hospital Privado, Cordoba, Argentina \\ ***Urology Department, Hospital Dos Servidores Do Estado, Rio De Janeiro,Brasil \\ ****Urology Department, Universidad De Unicamp, Campinas, Brasil \\ *****Urology Department, Fundaçao Felice Rosso, Belo Horizonte Brasil \\ ******Centro Medico Ultralitho, Florianopolis, Brazil
}

\section{Resumen}

Objetivo: Comunicar los resultados a largo plazo del estudio multicéntrico fase III del tratamiento de la incontinencia post- prostatectomía (IPP) con un Sling masculino ajustable (Argus $\left.{ }^{\circledR}\right)^{1}$.

Pacientes y métodos: 48 pacientes con IPP, 39 post radical y 9 post adenomectomía, ingresaron entre abril de 2003 y septiembre de 2004. 19 usaban 5 paños por día (3-8) pesando 83 gr. (17-198) por paño. 29 usaban clamp peniano o colector externo. El seguimiento promedio fue de 45 meses (36 -54). La edad fue de 67años (52-77). Fueron evaluados con el cuestionario ICIQ-SF y la calificación de seco (sin paños), mejora ( 1 paño) y falla ( 2 o más paños en 24 hs), incluyéndose a los slings extraídos. El Argus se implantó por vía perineal, transfiriéndolo con agujas al abdomen para ser ajustado y fijado con arandelas. El ajuste se realizó por presión uretral retrograda (PUR) entre 45 y $55 \mathrm{~cm}$ de agua.

Resultados: Evaluamos 47 de 48 pacientes (1, falleció antes de 3 años) resultando secos, mejorados y fallados, 31(66\%), 6 (12.8\%) y 10 (21\%) respectivamente. ICIQ-SF cambió de 19,5 a 6 (1-21). 5 de 31 secos, debieron reajustarse una vez. Fallaron 10 pacientes: 9 Argus retirados: $6(12,8 \%)$ por erosión, $3(6,2 \%)$ por infección y 1 usa 3 paños diarios. Hubieron 6 erosiones: 4 uretrales, 1 vesical y 1 parietal abdominal. El dolor persistió en 2 casos. Las complicaciones menores se resolvieron espontáneamente antes de 2 meses.

Conclusiones: El Argus $®$ demostró ser eficaz en el control de la IPP en el largo plazo en casi el 80\% de los pacientes evaluados. La infección y la erosión fueron las complicaciones más importantes y los desafíos a resolver.

Palabras clave: Swing masculino ajustable. Incontinencia masculina. Incontinencia pos prostatectomía.

\section{Long term results of a phase III multicentre trial of the adjustable male sling for treating urinary incontinence after prostatectomy: minimum 3 years}

\begin{abstract}
Objective: To communicate long- term results of the multicentre phase III trial post-prostatectomy urinary incontinence (PPI) treated with an adjustable male sling ${ }^{1}$.

Patients and Methods: 48 PPI patients were included in this trial from april 2003 to september 2004. 39 post radical prostatectomy and 9 post adenomectomy. 19 wore 5 pads per day (3-8) each weight 83gr (17-198). 29 wore condom catheter or penile clamp The Argus was implanted through perineal approach. Using needles, the sling was transferred to the abdominal wall where it was adjusted by washers. The adjustment was done with retrograde urethral pressure from 45 to $55 \mathrm{~cm}$ water. Clinical data were updated till September 2007. The mean follow-up was 45 months (36-54) and median age was 67 years (52-77). The evaluation was: the ICIQ-SF score and qualification as Dry: no pads, Improved: 1 pad and Failed: 2 or more pads in 24 hr, including those with slings removed.

Results: 47 were evaluated, resulting: 31 (66\%) Dry, 6 (12.8\%) Improved and 10 (21\%) Failed. The ICIQ-SF score changed from 19.5 to 6 . Of the 31 dry pts, 5 required one adjustment. 10 pts failed, 9 after sling removal, 6 due to erosion and 3 for infection. One patient failed with the sling in place, 6 erosions were registered: 4 in the urethra, 1 into the bladder and 1 through the abdominal wall. Perineal pain persisted in 2. One patient was excluded, died in September 2006.
\end{abstract}

Conclusions: Argus ${ }^{\circledR}$ has demonstrated its efficacy in long- term follow-up. The social continence rate was about $80 \%$. The important complication was erosion or infection.

Keywords: Adjustable male sling. Male incontinence. Incontinence pos-prostatectomy. 
$\mathrm{E}^{\mathrm{n}}$ los últimos 10 años el uso de un Sling bulbo uretral para el tratamiento de la incontinencia de orina pos prostatectomía despertó mucho interés como una alternativa frente a los otros métodos utilizados hasta entonces para tratarla. Sin duda fue el trabajo de Schaeffer et al. $^{2}$ el que entusiasmó a muchos investigadores (nosotros incluidos) a intentar este tipo de solución para un problema que sólo el esfínter urinario artificial había probado ser razonablemente eficiente en un numero importante de casos y sostenido a largo plazo ${ }^{3}$.

En abril de 2003 y hasta septiembre de 2004 incluimos a 48 pacientes incontinentes post prostatectomía en un estudio multicéntrico para ser tratados con un nuevo Sling ajustable de nuestro diseño (Argus ${ }^{\circledR}$ ) el cual, en nuestras manos, había demostrado resultados promisorios en un numero pequeño de pacientes . Los resultados a corto plazo del estudio multicéntrico fueron muy buenos en el $83 \%$ de los pacientes: $73 \%$ secos y $10 \%$ mejorados $^{1}$.

Esta serie fue madurando y los pacientes pudieron ser evaluados con un mínimo de 3 años, comprobando la consistencia y durabilidad de los resultados iniciales ya que se mantuvieron en el tiempo casi sin variantes.

A estos 48 pacientes, siguieron numerosos casos nuevos, alcanzando en la actualidad más de 1.500 Argus implantados en el mundo. Diversos centros siguieron nuestras indicaciones para su implante, siendo ya muchos los urólogos que han podido reproducir y aun mejorar nuestros resultados ${ }^{4-7}$.

La experiencia acumulada en estos años, nos ha permitido mejorar el diseño del Sling y con ello probablemente aumentar sus posibilidades de éxito en el tratamiento de esta devastadora condición, la incontinencia post prostatectomía.

\section{PACIENTES Y MÉTODOS}

Los datos de 47 de los 48 pacientes incontinentes que ingresaron al estudio en abril de 2003 fueron actualizados en todos los centros participantes a septiembre de 2007; 47 completaron sus datos hasta esta fecha, ya que 1 falleció, seco, en el 2006. El seguimiento promedio fue de 45 meses, con un mínimo de 36 y un máximo de 54 .

Todos los componentes del Sling Argus son de silicona de alta biocompatibilidad tisular.

El sistema es radiopaco permitiendo su control de posición en el postoperatorio. Está constituido por una almohadilla (pad) de espuma de siliconas para proveer una compresión acolchada de la uretra bulbar, que pende de 2 columnas con conos cuyo diseño permite su fijación por medio de arandelas contra la aponeurosis de la pared abdominal permitiendo así su ajuste y desajuste (Fig. 1). El Sling se implantó por una incisión perineal vertical que conserva a los músculos bulbo cavernosos in situ, contra los cuales se apoya la almohadilla o pad. Por fuera de estos se transfieren las columnas al abdomen por punción, con agujas especialmente diseñadas que ingresan a $1 \mathrm{~cm}$. por debajo y por fuera del borde inferior del pubis, rozando la rama isquiopubiana. El ajuste fue realizado por presión uretral retrograda de 45 a $55 \mathrm{~cm}$ de agua, con control endoscópico simultaneo de la oclusión uretral. ${ }^{1} \mathrm{El}$ uso del cistoscopio es obligado para descartar lesiones uretrovesicales con el paso de las agujas, permitiendo al tiempo del ajuste observar el sitio y el aspecto que toma la uretra al ser comprimida. La

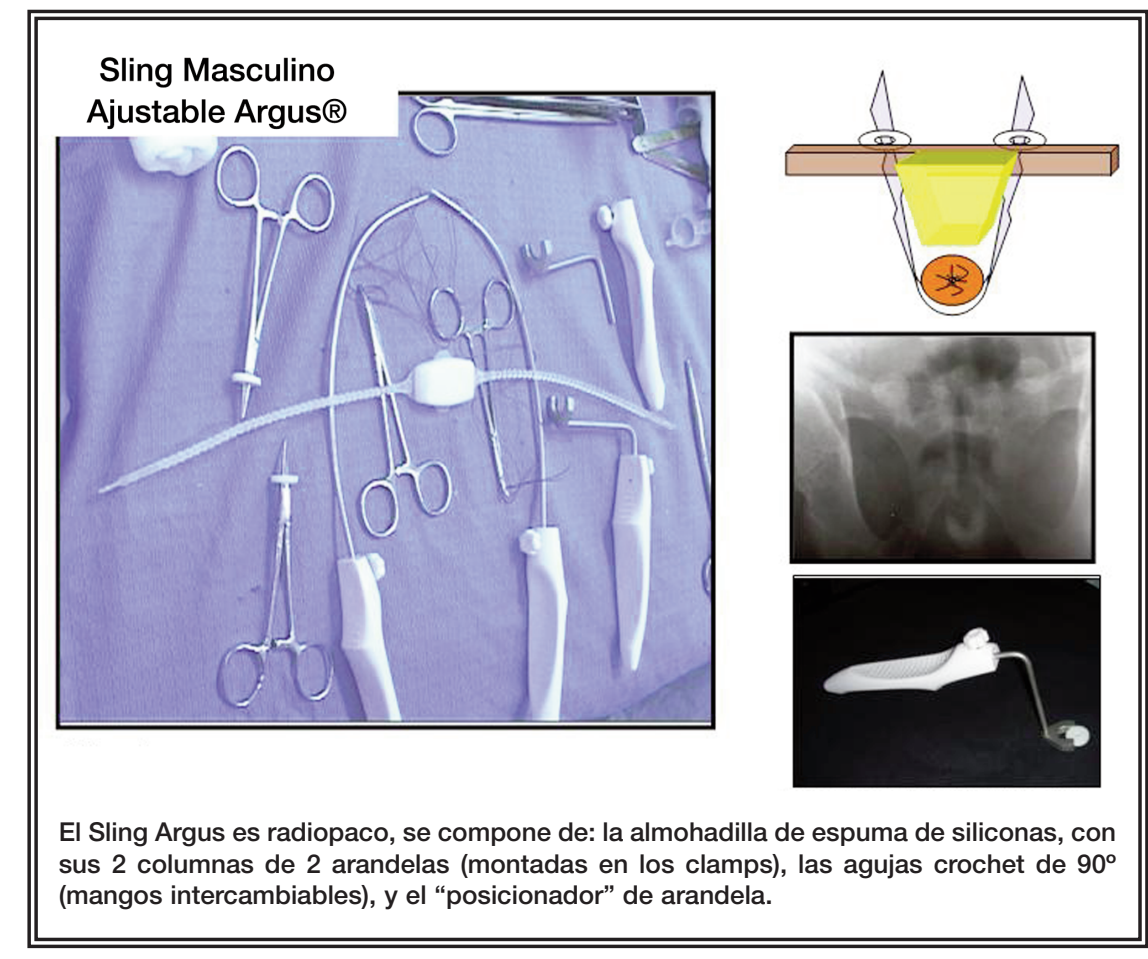

FIGURA 1. Componentes del Sistema Argus®. 
sonda uretral se retira a las 24 ó 48 h, y luego de comprobar la micción espontánea y el residuo, se externa al paciente.

Todos los pacientes completaron el cuestionario (ICIQ-SF) y se evaluó el estatus de continencia de acuerdo al uso diario de pañales y su peso. Se expresaron los resultados según no necesitaran paños (seco), 1 paño por día (mejorado) y 2 o más paños diarios (fallados) incluyendo en este ultimo grupo a los pacientes que les fuera retirado el Sling, por infección o erosión.

\section{RESULTADOS}

En total de los 47 pacientes permanecieron secos 31 (66\%), mejorados $6(12,8 \%)$ y fallaron $10(21 \%)$.

El ICIQ-SF descendió de 19,5 a 6 (21-0).

A 9 de los 10 pacientes considerados fallados, se les retiro el Sling por erosión (6) o por infección (3). El restante, con el Sling "in situ" usa 3 paños diarios.

1 paciente obeso (AN) que estuvo seco, se reajusto a los 30 meses de su implante al reiniciar su incontinencia como consecuencia de la perdida del $30 \%$ de su peso, manteniéndose seco desde el reajuste hasta la fecha.

En cuanto a las erosiones 4 fueron uretrales y ocurrieron el postoperatorio inmediato (antes de los 2 meses) y se comprobó su causa por rotación del pad presentando a la uretra un borde cortante.

1 paciente $(\mathrm{AD})$ Cuyo Sling fuera reemplazado a los 30 días de implantado por rotura de la columna, presentó pérdidas progresivas de orina luego de 3 años de estar seco, pasando a mejorado (utilizando 1 paño diario). El Sling se aflojó porque la arandela perforó (erosionó) la pared abdominal con desplazamiento lateral del Pad; actualmente espera para su recambio (Fig. 2) 1 paciente con perforación de la columna en vejiga (erosión/perforación inadvertida?) requirió del retiro del Sling.

1 paciente evoluciono seco por 5 meses postoperatorios pero con infección de la herida suprapúbica que a pesar del tratamiento prolongado con antibióticos y antisépticos locales finalmente obligo al retiro del sling por extrusión suprapúbica de las columnas.

1 paciente (JR) que fuera reajustado a los 4 meses de su implante, se mantuvo seco por 2 años con el Sling en posición, hasta su deceso en septiembre de 2006 y al no cumplir con los 3 años mínimos de seguimiento fue retirado del análisis los resultados.

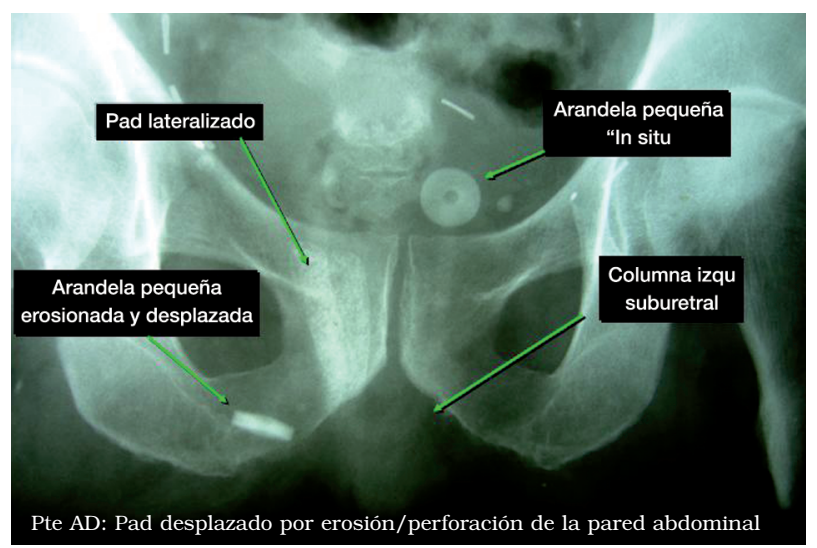

FIGURA 2. Rx simple del paciente AD: desplazamiento del pad a la derecha por la perforación de la pared abdominal por la arandela pequeña izquierda.

\section{DISCUSIÓN}

Del análisis de los resultados obtenidos del estudio multicéntrico de fase III y de la experiencia acumulada en 7 años consecutivos implantando el Sling ajustable para el tratamiento de la incontinencia post prostatectomía podemos afirmar que el Argus representa un recurso terapéutico validado, con resultados muy satisfactorios y durables para el control de esta desgraciada condición.

Nosotros estamos convencidos que el aumento pasivo (mínimo necesario) de la presión intrauretral, que permita a la mucosa su coaptación, es el principal, sino el único, mecanismo que explica el funcionamiento de los Sling bulbo uretrales, tal como lo expusiera su precursor, el Dr. JJ kaufman en sus publicaciones $^{8-10}$ y que luego fuera corroborado por Schaeffer et al. entre otros ${ }^{2,7-12}$. Por consiguiente creemos que no deberíamos considerar fallados a los pacientes que se les retiro el Sling, ya que al no mantenerse la compresión de la uretra, el regreso de la incontinencia es obligado, tal como lo comprobáramos en todos estos casos.

La posibilidad del sistema Argus de ser ajustado consiguiendo presiones intrauretrales regulables, conocidas y reproducibles ${ }^{11}$ así como su reajustabilidad postoperatoria, hace de este Sling un instrumento de gran utilidad; de hecho 5 (15\%) de los 31 pacientes consiguieron la continencia luego de un reajuste postoperatorio del Argus. Siguiendo con este concepto cabe destacar que en el comercio existen hasta hoy varios Slings pubo uretrales de los cuales el único ajustable (además del Argus) es el Remex ${ }^{\circledR}$ el cual valiéndose de un inteligente dispositivo (el Varitensor) suprapubico 
permite en el postoperatorio ajustar la tensión del sling necesaria a cada paciente para conseguir continencia con la menor tensión. Este sistema no obstante adolece del problema de poseer una cincha suburetral de polipropileno demasiado delgada y enrollable semejando a un cordel, lo cual, a nuestro entender lo hace proclive a las erosiones uretrales. das al sistema Argus, producto de nuestra experiencia, y que permiten mejorar su funcionamiento (Fig. 3) como la colocación de una arandela adicional de mayor diámetro (35 Mm.) sobre la aponeurosis contra la que apoya la pequeña original de ajuste, protegiendo a la pared abdominal de la erosión (Fig. 4); el refuerzo de las columnas, con lo que se dificulta

No obstante sus resultados a corto plazo son muy alentadores $^{14}$. Los slings no ajustables incluyen los fijados por tornillos a las ramas isquiopubianas (In Vance $\left.{ }^{\circledR}\right)^{15}$ y aunque sus investigadores lo recomiendan para la incontinencia leve, el hecho de no permitir su ajuste lo coloca francamente en desventaja. Lo mismo sucede con el Sling transobturatorio (Advance ${ }^{\circledR}$ ) el cual esta propuesto por sus investigadores para la incontinencia leve a moderada y el fundamento de su accionar sería el reposicionamiento intra abdominal del rabdoesfinter. Sin embargo al ser necesario tensionar de los extremos del sling para conseguir la continencia, no es posible descartar la contribución que efectúa la compresión uretral simultánea a la continencia lograda. Por otra parte al no poder mantener la tensión (carece de fijación en sus extremos) hace sus resultados poco duraderos.

El resto de los slings publicados son de confección artesanal y sus bases funcionales radican en la compresión de la uretra bulbar. Sin duda el tiempo y el uso cada vez más frecuente de este recurso remozado, permitirán el perfeccionamiento de los mismos, mejorando los resultados y disminuyendo las complicaciones.

Quisiéramos señalar aquí algunas modificaciones realiza-

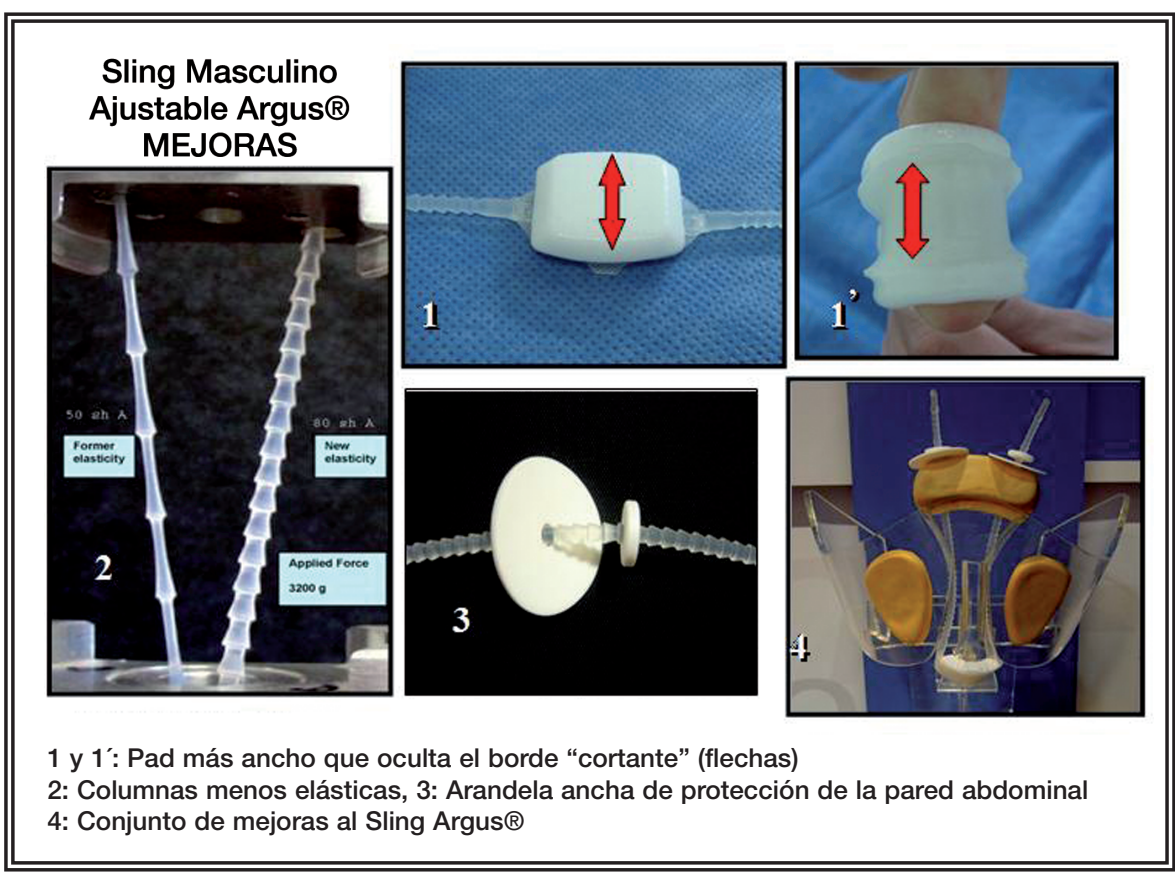

FIGURA 3. Mejoras introducidas al Sistema Argus $®$.

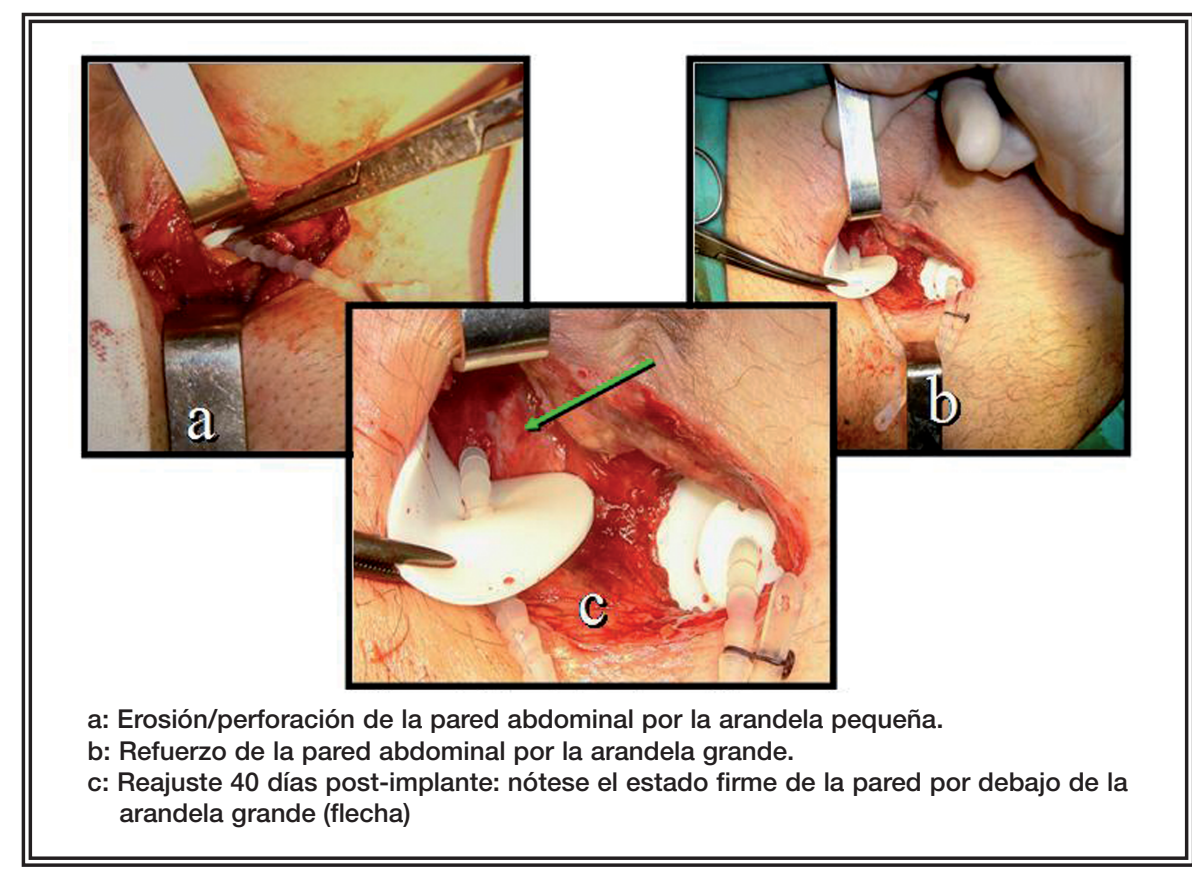

FIGURA 4. Arandelas grandes protegen a la pared abdominal de la erosión/perforación debida a la tensión ejercida y soportada por las arandelas pequeñas. 
el desajuste espontáneo a la vez que se evita que la columna continúe trabajando en el tiempo por su elasticidad y contribuya a la erosión; lo mismo sucede con el Pad de $0.5 \mathrm{~cm}$ más ancho, que supera por encima el borde de la cara posterior de la almohadilla protegiendo de este borde a la uretra y la posibilidad de sección o erosión de la misma (por rotación del Pad) como sucediera en los casos de erosión uretral; ya que en todos ellos, este borde se presentó contra la uretra, seccionándola en forma precoz entre los $15 \mathrm{y}$ 60 días del postoperatorio. El cuadro se caracterizó por un dolor perineal irreducible asociado a ardor y dolor miccional y la erosión fue diagnosticada por

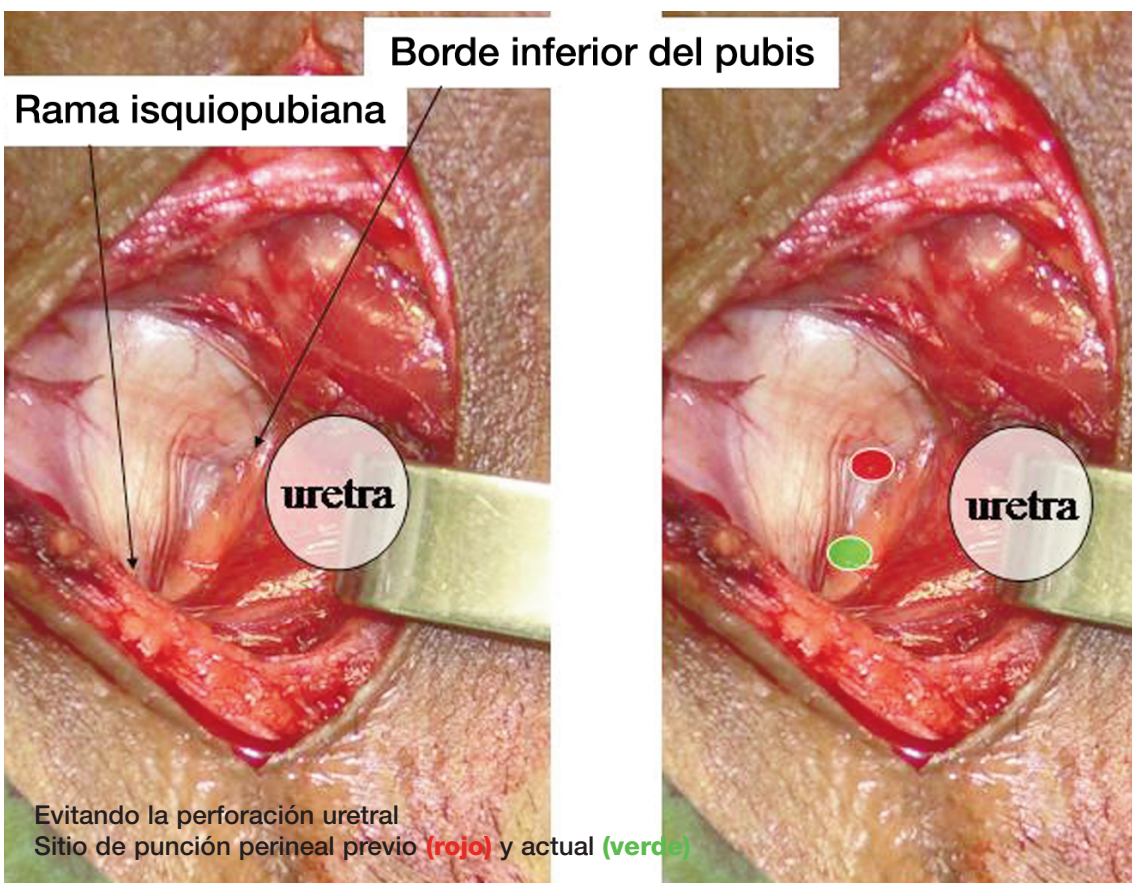

FIGURA 5. Sitio apropiado del paso perineal de la aguja para no lesionar la uretra: $1 \mathrm{~cm}$ por debajo y por fuera del ángulo isquiopubiano, rasando la rama isquiopubiana. el examen endoscópico. Cabe señalar que no ha habido repor-

tes de erosiones uretrales tardías hasta la fecha.

Realizamos además algunos finos ajustes de la técnica quirúrgica original, como el pasaje más bajo y afuera de la aguja (rasando la rama isquiopubiana) en su paso perineal con intención de evitar la perforación uretral (Fig. 5), y el descenso de la presión retrograda de ajuste operatoria a $35-40 \mathrm{~cm}$. de agua (en lugar de 45 a 55) para disminuir el dolor y la retención aguda postoperatorias manteniendo la continencia ${ }^{13}$.

Por otra parte, desde julio de 2007 un nuevo estudio multicéntrico ha comenzado para investigar la eficacia y seguridad de la colocación del Argus por vía transobturatoria, ya que los resultados inmediatos obtenidos con esta vía en 14 pacientes operados por uno de los investigadores (SVR) se mostraron muy alentadores.

\section{CONCLUSIONES}

El Sling ajustable Argus ${ }^{\circledR}$ demostró su eficacia en el control de la incontinencia post prostatectomía en el grupo de pacientes pertenecientes al estudio multicéntrico de fase III iniciado en abril del 2003, seguidos longitudinalmente en un tiempo promedio de 45 meses, con un mínimo de 3 años. Los resultados a largo plazo confirman y mantienen los exce- lentes resultados inicialmente comunicados, consiguiendo la continencia social en cerca del $80 \%$ de los pacientes evaluados (secos $66 \%+$ mejorados $12,8 \%=78,8 \%$ ).

En cuanto a las complicaciones cabe señalar que muchas de las que se observaron fueron aceptablemente bajas, de poca gravedad y de resolución espontánea, como el ardor miccional, la perforación uretral intraoperatoria, el dolor perineal, la retención aguda de orina, el edema y el adormecimiento perineo escrotal, mientras que aquellas más severas y que condujeron a las fallas por retiro del Sling fueron en la erosión en 6 pacientes $(12,8 \%$ ), ( 4 de ellas uretrales ) y la infección en 3 pacientes $(6,2 \%)$ siendo estas ultimas de presentación precoz. Ellas representan un motivo de constante preocupación para intentar evitarlas y resolverlas.

\section{REFERENCIAS}

1. Romano SV, Metrebian SE, Vaz F, Muller V, Levi D'Ancona CA, Costa De Souza EA, And Nakamura F. An adjustable male sling for treating urinary incontinence after prostatectomy - a phase iii multi-centre trial. BJU Int 2006; 97(3):533-539.

2. Shaeffer A, Clemens B, Ferrari M, Stamey T. The male bulbourethral. Sling procedure for post-radical prostatectomy incontinence. J Urol 1998;159:510-1515.

3. Tse V, Stone Ar. Incontinence after prostatectomy: the artificial urinay sphincter. BJU Int 2003;92(9)886-889. 
4. Urban M; Heracek J; Romano S V. New sugical method of treatement for male incontinence with argus adjustable male sling. Urologe Pro Praxi, 2006;1:20-23.

5. Gallistl H, Shlarp O, Hübner WA. Argus: Eine neuartige adjustierbare shlinge in der therapie der postprostatektomieincontinenz. Urologe A 45: 2006, Supl 1 S 43.

6. Sandoval JC, Salazar A, Oyanedel P, Verdugo F, Y Romano SV. Sling bulbouretral para el tratamiento de la incontinencia urinaria de esfuerzo masculina post cirugía prostática. Rev. Chilena De Urologia. 2007;72(2):185-188.

7. Reges R, Dàncona C, Reis L, Miyakoa R, Netto N. Treatmet of male urinary incontinence with male sling: long term follow-up Ics 2006; Abst 361.

8. Kaufman JJ. A New operation for male incontinence. Surg Gynecol Obstet. 1970;131(2): 295-299.

9. Kaufman JJ. Surgical treatment of post-prostatectomy incontinence: use of the penile crura to compress the bulbous urethra J Urol 1972;107(2):293-297.

10. Kaufman JJ. Treatment of post-prostatectomy urinary incontinence using a silicone gel prosthesis. Br J Urol. 1973;45(6): 646-653.

11. Comiter CV, Sullivan MP, Yalla SV. Retrograde leak point pressure for evaluating post-radical prostatectomy incontinence. Urology. 1997;49(2):231-236.
12. Stamey T. Perineal compression of the corpum spongiosum of the bulbar urethra. An operation for post-radical prostatectomy incontinence. J Urol(Part 2)1994;151:490A, Abstract 1049.

13. Romano SV . Técnica quirúrgica del sling masculino. Rev. Arg. De Urol. 2005;70(3).

14. Sousa-Escandon A, Rodriguez Gomez JI, Uribarri Gonzalez C And Marquez-Queimadelos A. Externally readjustable sling on male stress urinary incontinence treatment: point of technique and preliminary results. J Endourol. 2004;18(1):113-118.

15. Madjar S, Jacoby K, Giberti C Et Al. Bone anchored sling for the treatment of post-prostatectomy incontinence. $J$ Urol $2001 ; 165(1): 72-76$.

Correspondencia autor: Dr. Salomon V. Romano

Departamento de Urología.

Hospital Durand.

Díaz Vélez 5044. Parque Centenario,

Buenos Aires. Argentina

Tel.: 49825555

E-mail autor: sromano1@arnet.com.ar

Información artículo: Original - Incontinencia urinaria

Trabajo recibido: julio 2008

Trabajo aceptado: octubre 2008 